\title{
Performance Analysis of ATM Multiplexer with Bernoulli Traffic Sources
}

\author{
SHU-YIN CHIANG* \\ Department of Information and Telecommunication Engineering, Ming-Chuan University, \\ 5 Deb-Ming Rd. Gwei-Shan, Taoyuan 333, Taiwan, R.O.C.
}

(Received 29 July 2002)

In this paper, we study the simplified models of the ATM (Asynchronous Transfer Mode) multiplexer network with Bernoulli random traffic sources. Based on the model, the performance measures are analyzed by the different output service schemes.

Key words: ATM multiplexer; Bernoulli model

\section{INTRODUCTION}

In the context of switching networks, a multiplexer is a switching device whose purpose is to share an output link among a number of traffic sources. Multiplexers are characterized by their storage capacity (buffer length) and their processing capacity (data units per time slot), while traffic sources are characterized by arriving traffic models. Arriving traffic models are usually stochastic, and include the Poisson process in the continuous-time domain, the Bernoulli process in the discrete-time domain, the on/off traffic model with the on-periods and the off-periods exponentially distributed in the continuous-time domain or geometrically distributed in the discrete-time domain, etc.[1-3].

The particular multiplexer focused on in this paper is the ATM (Asynchronous Transfer Mode) multiplexer. The ATM multiplexer serves sources which generate ATM cells, each cell having a fixed length of 53 bytes. Although not covered in detail in this paper, the techniques developed here can also be applied to the so-called ATM switching element, which is similar to the ATM multiplexer except, instead of having one output link, sources are shared among multiple output links [3,4]. There are widely available models of the ATM multiplexer and the ATM switching element in the literature. The following model, which appears in, for example, [3,5-8], is used here: Each of the two systems under consideration serves $K$ sources. In the multiplexer case, there is one output buffer, and, in the switching element case, there are $M$ output buffers. For convenience, the time axis is slotted, with the slot duration equal to the cycle time (i.e., the time necessary to transmit one ATM cell). It is assumed that the sources are of a burst nature, which can be described mathematically by an on/off-type of model: during any particular time slot, a source is "on" (an active state) if it generates exactly one ATM cell, and the source is "off" (a passive state) if it does not generate any ATM cells. The transitions between these on and off states are characterized by

\footnotetext{
* Corresponding author. E-mail: sychiang@mcu.edu.tw
} 
two numbers, $0 \leq \alpha_{i} \leq 1$ and $0 \leq \beta_{i} \leq 1, i=1, \ldots, K$, where $\alpha_{i}$ is the conditional probability that the $i$ th sources is off during a time slot, given that it was on at the beginning of the time slot, and $\beta_{i}$ is the conditional probability that the $i$ th source is on during a time slot, given that it was off at the beginning of the time slot. It is assumed that no more than one ATM cell can be generated by each source in any time slot. For simplicity, each output buffer is assumed to have an unlimited storage capacity for ATM cells.

Using the above multiplexer and switch models, the main problem considered in the literature is to compute the mean buffer occupancy $(M B O)$ and the cell delay $(C D)$ as functions of $\alpha_{i}, \beta_{i}$, and $K$. The mean buffer occupancy and cell delay are useful performance measures because the former can be monitored to implement congestion control and flow control, and the latter is an important index of quality of service (QoS). Several numerical, results and bounds on these performance measures are already available in the literature (see, for instance, [3,5-8]); however, closed-form expressions for the performance measures are lacking. In addition, the cell loss rate $(C L R)$, a key performance measure for ATM networks [9-13], has been predicted by a heuristic approach, from the tail distribution of buffer occupancies of a buffer with infinity, capacity has not been analyzed at all. This is a consequence of the assumption that each output buffer has an unlimited storage capacity, i.e., since the buffer capacity is unlimited, the buffer cannot reach its maximum storing capacity even if a large number of sources are in the active state, making it impossible to obtain exact cell losses.

In this paper, we develop, using a simplified ATM multiplexer model, closed-form expressions for the three performance measures mentioned above and another important performance measure - throughput $(T P)$. We consider both priority and non-priority rules for serving by the ATM multiplexer. All sources considered in this paper are characterized by the Bernoulli model, that is, in every time slot, the source will generate an ATM cell with probability $p_{i}, i=l, \ldots, K$, and fail to do so with probability $1-p_{i}$. There are two advantages of reducing the two-parameter source description $\left(\alpha_{i}, \beta_{i}\right)$ to the one-parameter description $\left(p_{i}\right)$ :

1 The model simplifies sufficiently that closed-form expressions can be developed.

2 In practice, information on $p_{i}$ is available, but information on $\left(\alpha_{i}, \beta_{i}\right)$ often is not available.

Indeed, in many instances, measured communication node data is in the form of the source's utilization (i.e., the proportion of time that the sources is busy transmitting an ATM cell); consequently, experimental data leads naturally to a $p_{i}$ model formulation, but is often insufficient for the formulation of a $\left(\alpha_{i}, \beta_{i}\right)$ model.

Usage of the Bernoulli model is often criticized because the model does not account for the burst phenomenon which is considered to be an important property of ATM networks. However, for the ATM multiplexer model considered in this paper, when the number of sources $(K)$ is more than one, and when the probability $p_{i}$ becomes large, it is possible for many ATM cells to arrive at the multiplexer at the same time slot. In this sense, the burst phenomenon is partially captured by our model. It should be noted that a model analogous to the $p_{i}$ model described here was introduced in [14]; however, only a numerical solution for its analysis has been presented.

In summary, this paper considers an ATM multiplexer with random traffic sources based on the Bernoulli model and develops an analytical theory for performance analysis, including the analysis of $T P, C L R, M B O$, and $C D$.

The remainder of the paper is structured as follows. In Section 2, a detailed model of the ATM multiplexer is introduced and the problems are formulated. The performance analyses with priority and non-priority schemes are presented in Sections 3 and 4, respectively. Finally, conclusions are given in Section 5. 


\section{MODELING AND PROBLEM FORMULATION}

\subsection{Assumptions}

Consider an ATM multiplexer defined by the following assumptions.

The Multiplexer:

(I) The ATM multiplexer as shown in Figure 1, consisting of one output buffer and one output serve, which serve $K$ input random traffic sources.

(II) Synchronous transmission with a cycle time $T_{c}$ is used on both the input sources and the output server of the multiplexer, i.e., the time axis is slotted with the slot duration $T_{c}$ and sources and the output server begin transmitting ATM cells at the beginning of each time slot.

(III) The output buffer is characterized by its capacity $N<\infty$.

(IV) The output server with perfect reliability transmits one ATM cell during each time slot.

The Input Traffic:

(V) Each traffic sources, $S_{i}, i=t, \ldots, K$, generates one ATM cell during a time slot (i.e., in the active state) with probability $p_{i}, i=1, \ldots, K$, and is idle during a time slot (i.e., in the passive state) with probability $1-p_{i}, i=1, \ldots, K$.

The Output Buffer Service Schemes:

(VI) (a) (Priority service assumption) All sources have different priorities. Source 1 has the highest priority, source 2 has the second highest and so on, Let $d(t)$ denote the number of sources which are active at time slot $t$. Let $x(t-1)$ denote the number of ATM cells waiting to be transmitted in the output buffer at time slot $t-1$, Then $N-x(t-1)+1$, where " 1 " indicates that one ATM cell will transmit at time slot $t$ so that one unit of capacity is available for the input sources, is the available capacity of the output buffer at the beginning of time slot $t$. All of the ATM cells generated by the $d(t)$ sources are accepted by the output buffer for transmitting at the same time slot if $d(t) \leq N-x(t-1)+1$. Only the $N-x(t-1)+1$ ATM cells generated by the sources with higher priorities are accepted by the output buffer, and $d(t)-[N-x(t-1)+1]$ ATM cells from lower priority sources are lost if $d(t)>N-x(t-1)+1$.

(b) (Non-priority service assumption) All sources have equal priorities. The total number of active sources $d(t)$ and the occupancy of the buffer $x(t-1)$ are defined as in assumption

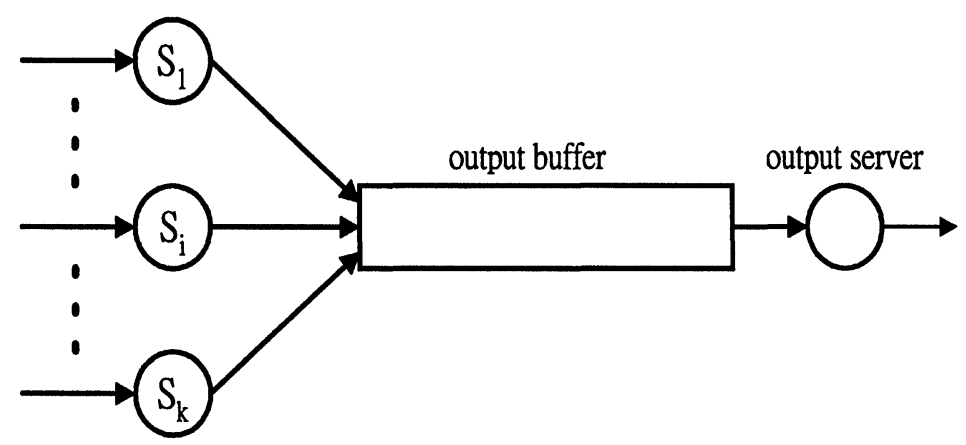

FIGURE 1 ATM multiplexer. 
(VI) (a) If $d(t) \leq N-x(t-1)+1$, then all of the ATM cells generated by the $d(t)$ sources are accepted by the output buffer at the same time slot. If $d(t)>N-x(t-1)+1$, then each ATM cell generated by the $d(t)$ sources is accepted by the output buffer with probability $(N-x(t-1)+1) / d(t)$, and is lost with probability $1-(N-x(t-1)+1) / d(t)$.

\subsection{Performance Measures}

The definitions of the four major performance measures considered in this paper are as follows:

1 The throughput $(T P)$ of the ATM multiplexer is the average steady state number of ATM cells transmitted by the output server in a time slot. It is the probability that the output server is not starved in a time slot.

2 The cell loss rate (CLR) of a source is the average steady state number of ATM cells generated by the sources and discarded by the output buffer per time slot. The total cell loss rate is the summation of the cell loss rates of all sources. Let $C L \mathrm{R}_{i}, i=1, \ldots, K$, denote the probabilities of the cell loss rate of sources $S_{i}$, and $C L R$ denote the total cell loss rates of all sources.

3 The mean buffer occupancy $(M B O)$ is the average steady state number of ATM cells found in the output buffer.

4 The cell delay $(C D)$ is defined as the number of time slots between the beginning of the arrival time slot and the beginning of the time slot during which the cell is transmitted and thus leaves the output buffer.

\subsection{Problems}

The goal of this paper is to derive closed-form solutions for the performance measures mentioned above in the ATM multiplexer networks (I)-(VI)(a) or (I)-(VI)(b). Since we do not find a solution for the network with arbitrary number of sources, only the solutions of the networks with $K=2$ are derived. The problems, then, addressed in this paper are:

Problem 1 Given model (I)-(VI)(a), derive explicit expressions for the solutions of TP, CLR, $M B O$, and $C D$.

Problem 2 Given model (I)-(VI)(b), derive explicit expressions for the solutions of TP, CLR, $M B O$, and $C D$.

Solutions of Problems 1 and 2 with $K=2$ are given in Sections 3 and 4, respectively. An extension to the cases of $K>2$ case is a subject of the future work.

\section{PERFORMANCE ANALYSIS WITH PRIORITY}

Let $X_{K}(j, s)$ denote the probability that the output buffer contains $j$ parts at time $s$ in the ATM multiplexer network (I)-(VI)(a) with $K$ sources. The dynamic, of buffer occupancy can be 
described by an irreducible, ergodic Markov chain with states $0,1, \ldots, N$. Since the Markov chain is irreducible and ergodic, it converges to a unique equilibrium distribution. Let

$$
X_{K}(j)=\lim _{s \rightarrow \infty} X_{K}(j, s), \quad 0 \leq j \leq N
$$

It is useful to observe the resulting transition diagram of the Markov chain (see Fig. 2). The values $G_{0}, G_{1}, G_{2}$ in Figure 2 represent the total probability of no sources being active (i.e., $\left.G_{0}=\left(1-p_{1}\right)\left(1-p_{2}\right)\right)$, one source being active (i.e., $\left.G_{1}=p_{1}\left(1-p_{2}\right)+p_{2}\left(1-p_{1}\right)\right)$, and both of sources being active (i.e., $\left.G_{2}=p_{1} p_{2}\right)$, respectively. Then, this equilibrium distribution must satisfy the following equilibrium equations of the Markov transition equation:

$$
\begin{aligned}
X_{2}(0)= & \left(1-p_{1}\right)\left(1-p_{2}\right) X_{2}(0)+\left(1-p_{1}\right)\left(1-p_{2}\right) X_{2}(1) \\
X_{2}(1)= & {\left[\left(1-p_{1}\right) p_{2}+\left(1-p_{2}\right) p_{1}\right] X_{2}(0) } \\
& +\left[\left(1-p_{1}\right) p_{2}+\left(1-p_{2}\right) p_{1}\right] X_{2}(1) \\
& +\left(1-p_{1}\right)\left(1-p_{2}\right) X_{2}(2) \\
X_{2}(2)= & p_{1} p_{2} X_{2}(0)+p_{1} p_{2} X_{2}(1) \\
& +\left[p_{1}\left(1-p_{2}\right)+p_{2}\left(1-p_{1}\right)\right] X_{2}(2) \\
& +\left(1-p_{1}\right)\left(1-p_{2}\right) X_{2}(3), \\
X_{2}(j)= & p_{1} p_{2} X_{2}(j-1)+\left[p_{1}\left(1-p_{2}\right)+p_{2}\left(1-p_{1}\right)\right] X_{2}(j) \\
& +\left(1-p_{1}\right)\left(1-p_{2}\right) X_{2}(j+1), \quad 3 \leq j \leq N-1, \\
X_{2}(N)= & p_{1} p_{2} X_{2}(N-1)+\left[1-\left(1-p_{1}\right)\left(1-p_{2}\right)\right] X_{2}(N) .
\end{aligned}
$$

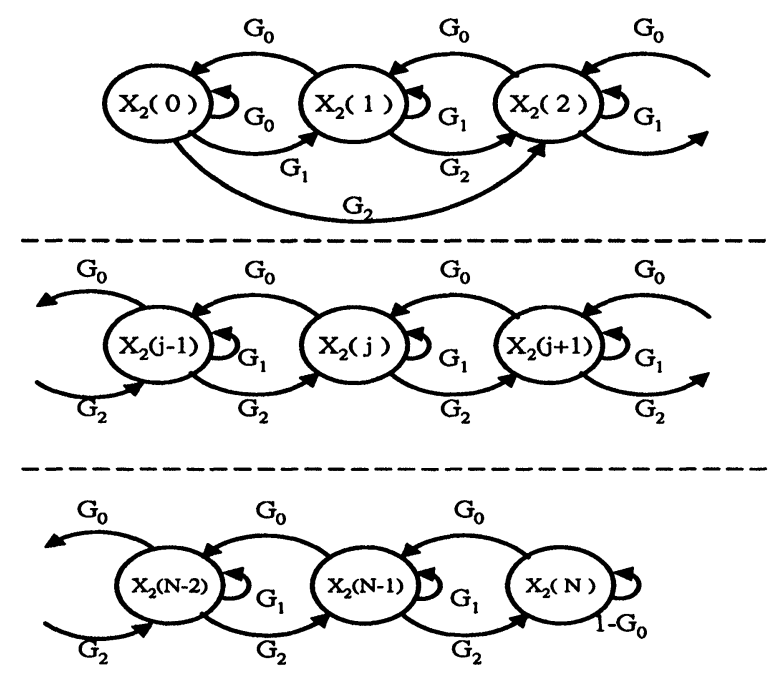

FIGURE 2 State transition diagram for Markov chain with two sources and buffer size $N$. 
Solving Eq. (3.2), we get

$$
\begin{aligned}
& X_{2}(1)=\frac{1-\left(1-p_{1}\right)\left(1-p_{2}\right)}{\left(1-p_{1}\right)\left(1-p_{2}\right)} X_{2}(0), \\
& X_{2}(j)=\frac{p_{1}^{j-1} p_{2}^{j-1}}{\left(1-p_{1}\right)^{j}\left(1-p_{2}\right)^{j}} X_{2}(0), \quad 2 \leq j \leq N .
\end{aligned}
$$

Thus, we have the steady-state probabilities $X_{2}(j)=0, \ldots, N$. Using the normalizing condition

$$
1=X_{2}(0)+X_{2}(1)+X_{2}(2)+\cdots+X_{2}(N)
$$

we have

$$
1=X_{2}(0)\left\{1+\left[\frac{1}{\left(1-p_{1}\right)\left(1-p_{2}\right)}-1\right]+\sum_{j=2}^{N} \frac{p_{1}^{j-1} p_{2}^{j-1}}{\left(1-p_{1}\right)^{j}\left(1-p_{2}\right)^{j}}\right\}
$$

Let $\alpha=p_{1} p_{2} /\left[\left(1-p_{1}\right)\left(1-p_{2}\right)\right]$. Then

$$
\begin{aligned}
1= & X_{2}(0)\left[\frac{1}{\left(1-p_{1}\right)\left(1-p_{2}\right)}+\frac{\alpha}{\left(1-p_{1}\right)\left(1-p_{2}\right)}\right. \\
& \left.+\frac{\alpha^{2}}{\left(1-p_{1}\right)\left(1-p_{2}\right)}+\cdots+\frac{\alpha^{N-1}}{\left(1-p_{1}\right)\left(1-p_{2}\right)}\right]
\end{aligned}
$$

Consequently, the expression for $X_{2}(0)$ is obtained as follows:

$$
X_{2}(0)= \begin{cases}\frac{\left(1-p_{1}\right)\left(1-p_{2}\right)(1-\alpha)}{11 \alpha^{N}}, & \alpha \neq 1 \\ \frac{\left(1-p_{1}\right)\left(1-p_{2}\right)}{N}, & \alpha=1\end{cases}
$$

Based on the definitions of $C L R_{i}, i=1, \ldots, K$ and $C L R$ given in Section 2, they can be evaluated below. According to the priority service scheme, the ATM cell generated by source $S_{1}$ is never discarded by the output buffer. Therefore, we have

$$
C L R_{1}=0
$$

If source $S_{1}$ has an ATM cell to transmit, and the output buffer is full at the beginning of a time slot, the ATM cell generated by source $S_{2}$ is not accepted by the output buffer at the beginning of this time slot. That is, source $S_{2}$ is blocked, and the cell from $S_{2}$ is lost. Since the probability of $S_{2}$ being blocked is equal to $p_{1} X_{2}(N)$, we obtain

$$
C L R_{2}=p_{2}\left[p_{1} X(N)\right]
$$


Using equation (3.3) and (3.7), we have

$$
C L R_{2}= \begin{cases}\frac{p_{1} p_{2}(1-\alpha) \alpha^{N-1}}{1-\alpha^{N}}, & \alpha \neq 1 \\ \frac{p_{1} p_{2}}{N}, & \alpha=1\end{cases}
$$

Based on (3.3), (3.7), (3.9), the $T P, C L R, M B O, C D$ of the network are obtained as follows: For the output device can transmit an ATM cell during a time slot, it must be not starved. Therefore, the throughput (TP), can be calculated as follows:

$$
T P=l-X_{2}(0)
$$

Using equation (3.7), we obtain

$$
T P= \begin{cases}\frac{\left(1-\alpha^{N}\right)-\left(1-p_{1}\right)\left(1-p_{2}\right)(1-\alpha)}{1-\alpha^{N}}, & \alpha \neq 1 \\ \frac{N-\left(1-p_{1}\right)\left(1-p_{2}\right)}{N}, & \alpha=1\end{cases}
$$

The total cell loss rate, $C L R$, can be calculated by using (3.8) and (3.9). Since

$$
C L R=\sum_{i=1}^{2} C L R_{i},
$$

we have

$$
C L R= \begin{cases}\frac{p_{1} p_{2}(1-\alpha) \alpha^{N}}{1-\alpha^{N}}, & \alpha \neq 1 \\ \frac{p_{1} p_{2}}{N}, & \alpha=1\end{cases}
$$

According to the definition of $M B O$ in Section 2, it can be expressed in terms of the steady state probabilities of buffer occupancy as follows:

$$
M B O=\sum_{i=0}^{N} i \cdot X_{2}(i)
$$

From Eqs. (3.3) and (3.7), we have

$$
M B O= \begin{cases}\frac{1-(1+N-\alpha N) \alpha^{N}-\left(1-p_{1}\right)\left(1-p_{2}\right)(1-\alpha)^{2}}{(1-\alpha)\left(1-\alpha^{N}\right)}, & \alpha \neq 1, \\ \frac{N(N+2)-2\left(1-p_{1}\right)\left(1-p_{2}\right)}{2 N}, & \alpha=1 .\end{cases}
$$

Using the expression for $M B O$, the $C D$ can be calculated by applying Little's Theorem. The average number of customers in the system in Little's Theorem is corresponding to $M B O$ in 
the multiplexer, the customer arrival rate is corresponding to $T P$, and the average customer waiting in queue is corresponding to $C D$. Therefore, the $C D$ is calculated as follows:

$$
\begin{aligned}
C D & =\frac{M B O}{T P}, \\
& = \begin{cases}\frac{\left[1-(1+N-\alpha N) \alpha^{N}-\left(1-p_{1}\right)\left(1-p_{2}\right)(1-\alpha)^{2}\right]\left(1-\alpha^{N}\right)}{(1-\alpha)\left(1-\alpha^{N}\right)\left[\left(1-\alpha^{N}\right)-\left(1-p_{1}\right)\left(1-p_{2}\right)(1-\alpha)\right]}, & \alpha \neq 1, \\
\frac{N(N+2)-2\left(1-p_{1}\right)\left(1-p_{2}\right)}{2\left[N-\left(1-p_{1}\right)\left(1-p_{2}\right)\right]}, & \alpha=1 .\end{cases}
\end{aligned}
$$

To show the relations between the performance measures and the ATM multiplexer parameters $\left(p_{i}, i=1, \ldots, K\right.$, and $\left.N\right)$, three cases are considered. For the first case, the total arrival rate $p_{1}+p_{2}$ is equal to 1 , and the buffer size is changed from 10 to 50 . It follows from Eq. (3.7), that the steady state probability of $X_{2}(0)$ is monotonically decreasing in $N$. Therefore, the throughput $T P$ in (3.11) is monotonically increasing in $N$. This result is illustrated in Figure 3(a). In Figure 3(b), we plot the relation between the total cell loss rate $C L R$ in (3.12) and the buffer size $N$. It is clear that the curve is monotonically decreasing with respect to buffer size $N$. When the buffer sizes goes to infinity, the $C L R$ will go to zero. The mean buffer occupancy $M B O$ in (3.13) and the cell delay $C D$ in (3.14) versus the buffer size $N$ are plotted in Figure 3(c) and 3(d), respectively. These figures show that $M B O$ and $C D$ are increasing in $N$.

For the second case, the input traffic probability of source $S_{1}$ is varied from 0 to 1 , and the buffer size is fixed. The TP and $C L R$ are increasing with respect to the input traffic probability of $S_{1}$ (i.e., $p_{1}$ as shown in Figs. 4(a) and 4(b). Similar conclusions can be obtained for the $M B O$ and $C D$, and their relations with respect to $p_{1}$ are shown in Figures $4(\mathrm{c})$ and $4(\mathrm{~d})$, respectively.

Finally, consider the third case $p_{1}+p_{2}$ is greater than 1 and $N$ is changed from 1 to 200 . Given, $p_{1}=p_{2}=0.7$, the relations between the throughput $T P$ and the output buffer size $N$, the cell loss rate $C L R$ and $N$, the mean buffer occupancy $M B O$ and $N$, and the cell delay $C D$
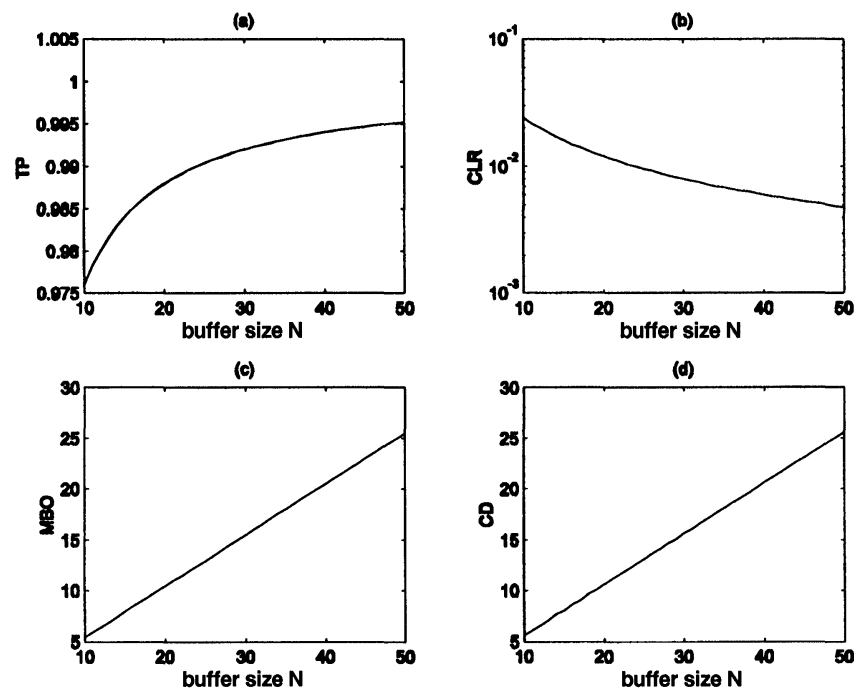

FIGURE 3 (a) Throughput TP versus buffer size $N$; (b) Total cell loss rate $C L R$ versus buffer size $N$; (c) Mean buffer occupancy $M B O$ versus buffer size $N$; (d) Cell delay $C D$ versus buffer size $N$; for $K=2, p_{1}=0.4$, and $p_{2}=0.6$. 

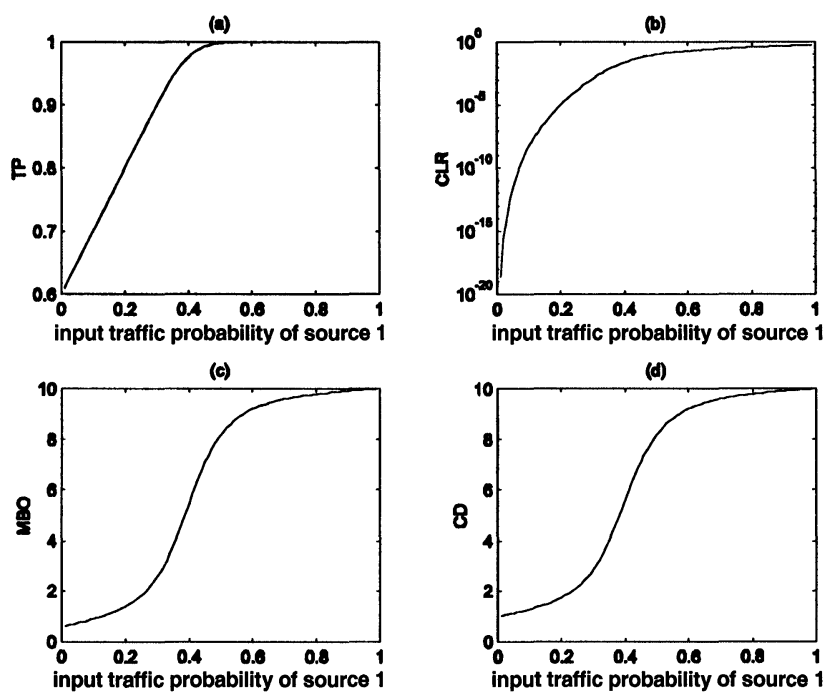

FIGURE 4 (a) Throughput TP versus input traffic probability of source $S_{1}$ (i.e., $p_{1}$ ); (b) Total cell loss rate $C L R$ versus input traffic of source $S_{1}$; (c) Mean buffer occupancy $M B O$ versus input traffic probability of source $S_{1}$; (d) Cell delay $C D$ versus input traffic probability of source $S_{1}$; for $K=2, N=10$, and $p_{2}=0.6$.

and $N$ are shown in Figures 5(a), 5(b), 5(c), and 5(d), respectively. Most of the relations are in agreement with out intuition expect the relation between $C L R$ and $N$. From Figure 5(b), as $N$ becomes large, the $C L R$ is approaching to 0.4. By out intuition, if the size of the output buffer is very large, the output buffer can accept as many ATM cells as it can. Hence, the CLR should be zero. This, however, is not true, as shown in Figure 5(b). The reason is that if the total cell arrival rate is always greater than the service rate of the output device, a large buffer is still possible to be full in the long term so that there is cell loss. Therefore, based on the
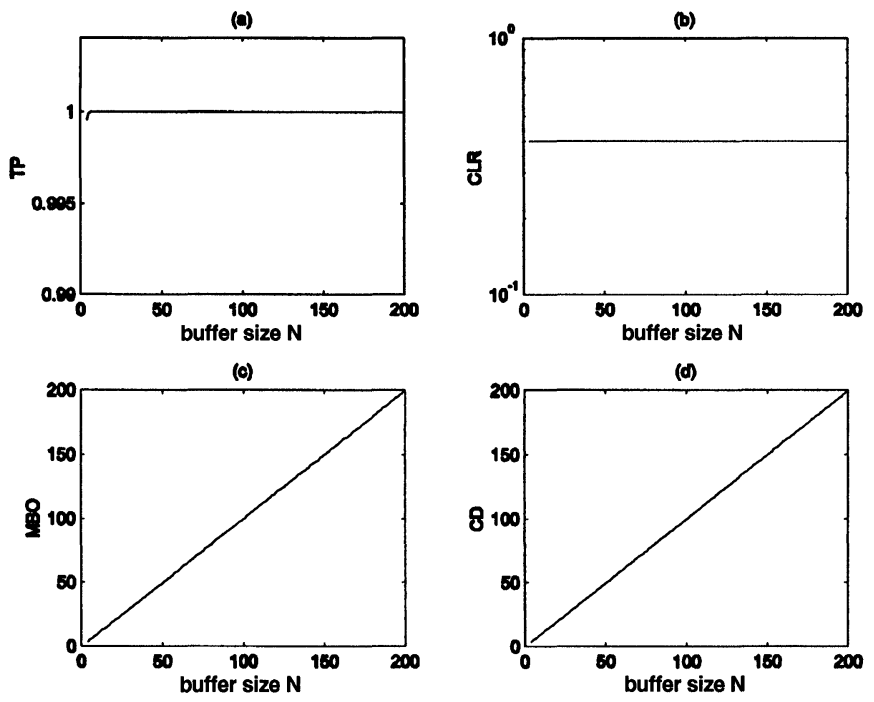

FIGURE 5 (a) Throughput TP versus buffer size $N$; (b) Total cell loss rate $C L R$ versus buffer size $N$; (c) Mean buffer occupancy $M B O$ versus buffer size $N$; (d) Cell delay $C D$ versus buffer size $N$; for $K=2, p_{1}=0.7$, and $p_{2}=0.7$. 
finding, we can learn the lesson that a large output buffer cannot eliminate the problem of cell loss. Also, as shown in Figure 5(d), a large buffer can create a long cell delay. This conclusion is useful for the design of the ATM multiplexer.

\section{PERFORMANCE ANALYSIS WITHOUT PRIORITY}

For the multiplexer with $K=2$, the equilibrium equations are derived as follows:

$$
\begin{aligned}
X_{2}(0)= & \left(1-p_{1}\right)\left(1-p_{2}\right) X_{2}(0)+\left(1-p_{1}\right)\left(1-p_{2}\right) X_{2}(1) \\
X_{2}(1)= & {\left[\left(1-p_{1}\right) p_{2}+\left(1-p_{2}\right) p_{1}\right] X_{2}(0) } \\
& +\left[\left(1-p_{1}\right) p_{2}+\left(1-p_{2}\right) p_{1}\right] X_{2}(1) \\
& +\left(1-p_{1}\right)\left(1-p_{2}\right) X_{2}(2) \\
X_{2}(2)= & \left(\frac{1}{2} p_{1} p_{2}+\frac{1}{2} p_{2} p_{1}\right) X_{2}(0)+\left(\frac{1}{2} p_{1} p_{2}+\frac{1}{2} p_{2} p_{1}\right) X_{2}(1) \\
& +\left[p_{1}\left(1-p_{2}\right)+p_{2}\left(1-p_{1}\right)\right] X_{2}(2) \\
& +\left(1-p_{1}\right)\left(1-p_{2}\right) X_{2}(3), \\
X_{2}(j)= & \left(\frac{1}{2} p_{1} p_{2}+\frac{1}{2} p_{2} p_{1}\right) X_{2}(j-1)+\left[p_{1}\left(1-p_{2}\right)+p_{2}\left(1-p_{1}\right)\right] X_{2}(j) \\
& +\left(1-p_{1}\right)\left(1-p_{2}\right) X_{2}(j+1), \quad 3 \leq j \leq N-1, \\
X_{2}(N)= & \left(\frac{1}{2} p_{1} p_{2}+\frac{1}{2} p_{2} p_{1}\right) X_{2}(N-1)+\left[1-\left(1-p_{1}\right)\left(1-p_{2}\right)\right] X_{2}(N) .
\end{aligned}
$$

Comparing (4.1) with (3.2), we find that they are identical. Therefore, the equivalence property is formulated in the following: The equilibrium equations with non-priority are exactly equal to the equilibrium equations with priority. That is, the steady state probabilities $X_{K}(j)$ based on the priority and non-priority assumptions, $j=0, \ldots, N, K=2$, are the same.

The above statement can be rationalized as follows: Since the output buffer only cares whether an ATM cell is coming or not, it does not care where the cell is coming from. Consequently, no matter whether the output buffer is using the priority serving scheme or the non-priority serving scheme, the output buffer accepts the cell, and the state of the network is changed from $x(t)$, defined in Section 2, to $x(t)+1$. Continue the same process for other states. Therefore, the equilibrium equations between the priority and the non-priority ATM multiplexers are the same.

It follows from the above that the $T P \mathrm{~s}, M B O \mathrm{~s}$, and $C D$ s of two kinds of multiplexers should be the same. The only different result between these two multiplexers are on the evaluation of $C L R_{i}, i=1, \ldots, K$. The next paragraph is devoted to derive the cell loss rate of each source with non-priority.

According to the non-priority service scheme, when an ATM cell generated by $S_{2}$ is accepted by the output buffer at the beginning of a time slot and the buffer is full at the beginning of the time slot and ATM cell generated by $S_{1}$ is lost. That is, $S_{1}$ is blocked, and the probability of $S_{1}$ being blocked is $(1 / 2) p_{2} X_{2}(N)$. We obtain

$$
C L R_{1}=p_{1}\left[\frac{1}{2} p_{2} X_{2}(N)\right] \text {. }
$$


Then, we have

$$
C L R_{1}= \begin{cases}\frac{1}{2} \frac{p_{1} p_{2}(1-\alpha) \alpha^{N-1}}{1-\alpha^{N}}, & \alpha \neq 1, \\ \frac{1}{2} \frac{p_{1} p_{2}}{N}, & \alpha=1 .\end{cases}
$$

If the situation is reversed, the source $S_{2}$ is blocked, and the cell from $S_{2}$ is lost. The probability of $S_{2}$ being blocked is $(1 / 2) p_{1} X_{2}(N)$. We obtain

$$
C L R_{2}=p_{2}\left[\frac{1}{2} p_{1} X_{2}(N)\right] \text {. }
$$

Then,

$$
C L R_{2}= \begin{cases}\frac{1}{2} \frac{p_{1} p_{2}(1-\alpha) \alpha^{N-1}}{1-\alpha^{N}}, & \alpha \neq 1, \\ \frac{1}{2} \frac{p_{1} p_{2}}{N}, & \alpha=1 .\end{cases}
$$

For the total cell loss rate $C L R$ of the multiplexer, it can be calculated by using (4.2) and (4.4) as follows:

$$
\begin{aligned}
C L R & =\sum_{i=1}^{2} C L R_{i} \\
& = \begin{cases}\frac{p_{1} p_{2}(1-\alpha) \alpha^{N-1}}{1-\alpha^{N}}, & \alpha \neq 1, \\
\frac{p_{1} p_{2}}{N}, & \alpha=1 .\end{cases}
\end{aligned}
$$

The expression of $C L R$ in the above equation is identical to that in Eq. (3.12). Moreover, from Eqs. (4.2) and (4.4), we have $C L R_{1}=C L R$.

Then, based on the results on $C L R_{i}, i=1, \ldots, K$, the four measures in the ATM multiplexer (I)-(VI)(b) can be formulated as follows: For the ATM multiplexer (I)-(VI)(b) with $K=2$, the throughput $T P$, the total cell loss rate $C L R$, the mean buffer occupancy $M B O$ and the cell delay $C D$ are the same as those in the ATM multiplexer (I)-(VI)(a) with $K=2$.

The reason for the result on CLR can be explained as follows: According to the conservation, the input flow of the output buffer should be equal to the output flow of the buffer in both of the two multiplexers (i.e., priority and non-priority). Hence, the multiplexer with the non-priority serving scheme suffers the same amount of cell loss as that with the priority serving scheme. Therefore, the two multiplexers have the same total CLRs.

\section{CONCLUSIONS}

A performance analysis of a communication network is considered by many as a necessary condition to implement a better QoS improvement project or to implement congestion control and flow control for existing networks or to achieve a cost-effect design for new networks. The theory developed in this paper provides an analysis of a basic element (i.e., an ATM multiplexer) in communication networks. This result can be used as a building block to analyze complicated communication networks. This paper has derived closed-form expressions of the following performance measures - the throughput, the cell loss rate, the mean buffer occupancy, and the cell delay - for the ATM multiplexers, where the serving schemes 
of the output buffer are based on the priority assumption or the non-priority assumption. These expressions can be used to evaluate the performance of the ATM multiplexer under consideration, and used as a tool to construct an ATM multiplexers in the design stage.

Although the performance analysis is quite complete for the multiplexer with number of sources being 2 , additional research is needed to analyze the network with arbitrary number of sources. It is the subject of the future work.

\section{References}

[1] Kobayshi, H. and Konheim, A. G. (1977) Queueing models for computer communications system analysis, IEEE Trans. Commun., COM-25(1), 2-29.

[2] Frost, V. S. and Melamed, B. (1994) Traffic modeling for telecommunications networks, IEEE Commun. Mag., 70-81.

[3] Bruneel, H. and Wittevrongel, S. (1996) An approximate analytical technique for the performance evaluation of ATM switching elements with burst routing, Computer Networks and ISDN Systems, 28, 325-343.

[4] Akyildiz, F., Joe, I., Fujimoto, R. M. and Nikolaidis, I. (1997) Parallel simulation of end-to-end ATM -models, Computer Networks and ISDN Systems, 29, 617-630.

[5] Toweley, D. and Wolf, J. K. (1979) On the statistical analysis of queue lengths and waiting times for statistical multiplexers with ARQ retransmission schemes, IEEE Trans. Commun., COM-27(4), 693-702.

[6] Burneel, H. (1987) On discrete buffers in a two-state environment, IEEE Trans. Commun., COM-25(1), 32-38.

[7] Burneel, H. (1988) Queueing behavior of statistical multiplexers with correlated inputs, IEEE Trans. Commun., COM-36(12), 1339-1341.

[8] Wu, J. S. and Kuo, F. J. (1997) Bounds on waiting time for multiplexing leaky buckets enforced sources in ATM networks, IEE Proc.-Commun., 144(1), 17-23.

[9] Bae, J. J. and Suda, T. (1991) Survey of traffic control schemes and protocols in ATM networks, Proceedings of the IEEE, 79(2), 170-189.

[10] Jam, R. (1996) Congestion control and traffic management in ATM networks: Recent advances and a survey, Computer Networks and ISDN Systems, 28, 1723-1738.

[11] Lee, N. H., Mun, Y. S. and Kim, B. G. (1997) Performance analysis of delay-loss priority mechanism using Markov modulated arrival stream, IEE Proc.-Commun, 144(5), 311-315.

[12] Roginsky, A. L., Tomek, L. A. and Christensen, K. J. (1997) Analysis of ATM cell loss for systems I with on/off traffic sources, IEE Proc.-Commun., 144(3), 129-134.

[13] Chowdhury, S. and Sengupta, B. (1997) Design and performance of a threshold-based load balancing scheme in an ATM switch, Computer Networks and ISDN System, 29, 157-164.

[14] Janakiraman, N., Pagurek, B. and Neilson, J. B. (1980) Multiplexing low-speed buffered data terminals, IEEE Trans. Commun., COM-28(10), 1838-1843. 


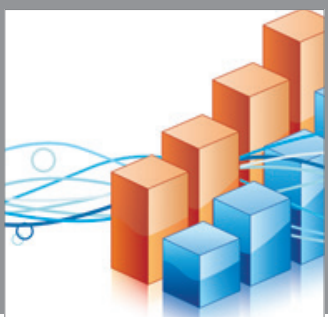

Advances in

Operations Research

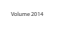

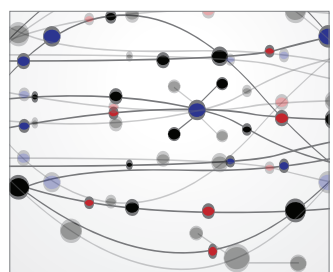

\section{The Scientific} World Journal
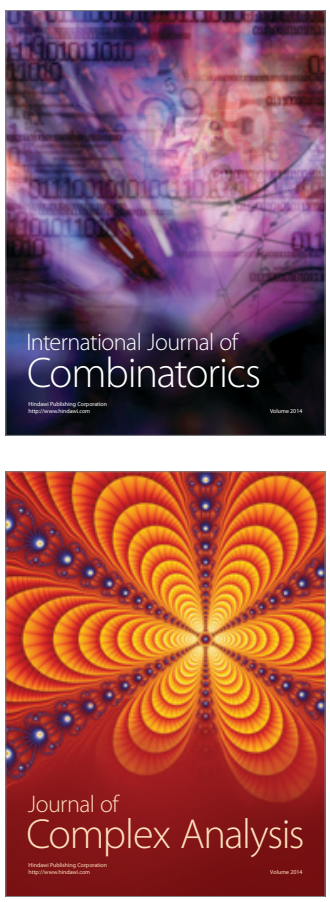

International Journal of

Mathematics and

Mathematical

Sciences
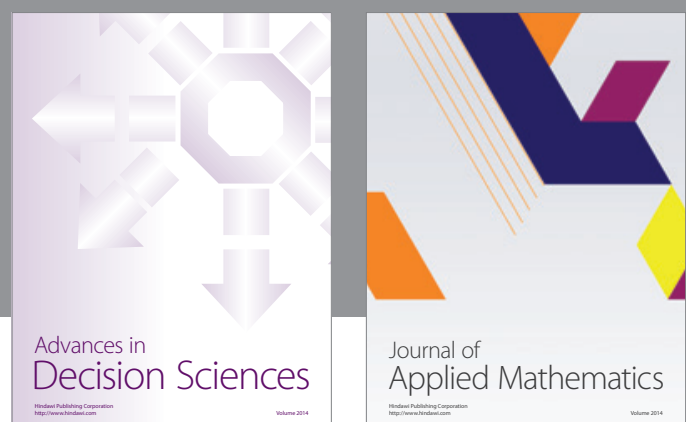

Journal of

Applied Mathematics
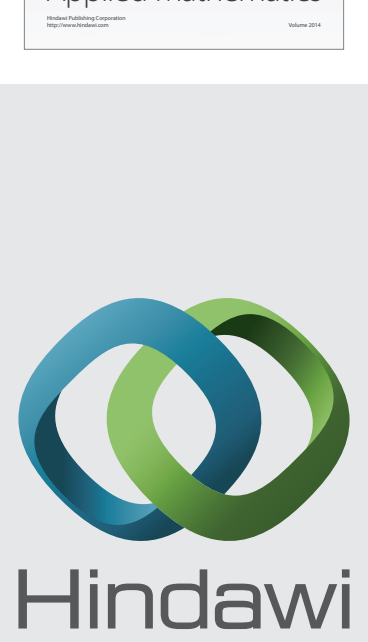

Submit your manuscripts at http://www.hindawi.com
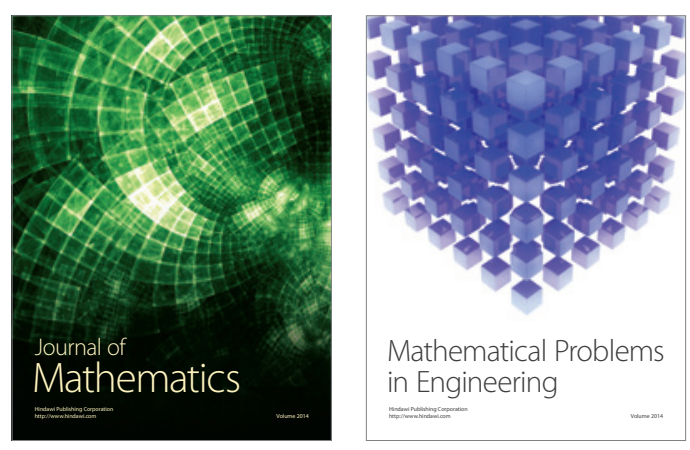

Mathematical Problems in Engineering
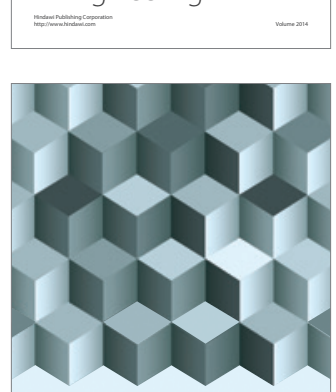

Journal of

Function Spaces
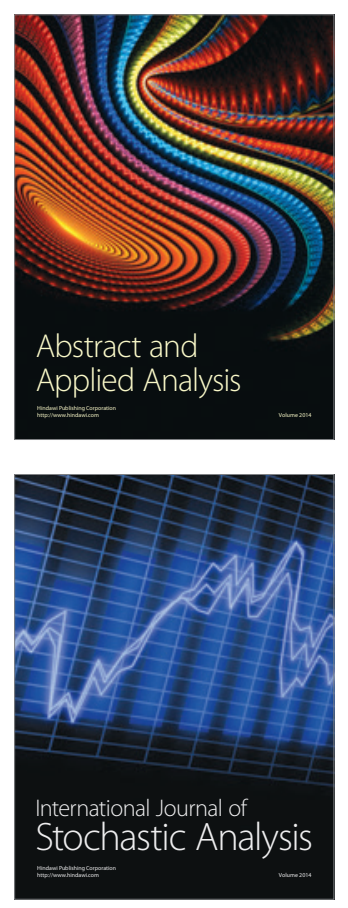

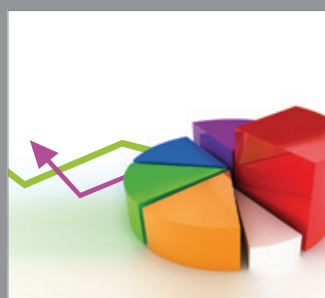

ournal of

Probability and Statistics

Promensencen
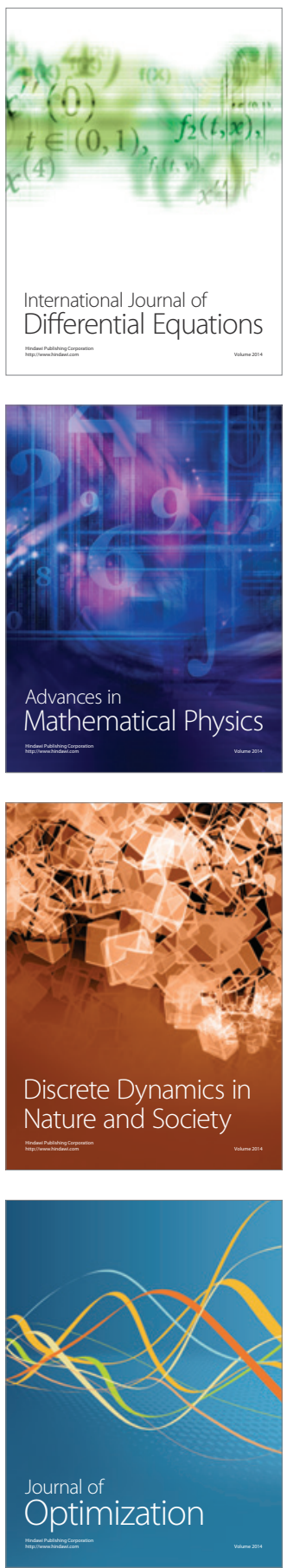